\title{
Altered Structural and Functional Connectivity of Juvenile Myoclonic Epilepsy: An fMRI Study
}

\author{
Chengqing Zhong $\mathbb{D}^{1,},{ }^{1,2}$ Rong Liu, ${ }^{2}$ Cheng Luo $\mathbb{D},{ }^{3}$ Sisi Jiang, ${ }^{3}$ Li Dong, ${ }^{3}$ Rui Peng, ${ }^{3}$ \\ Fuqiang Guo $\mathbb{D}^{1,4}$ and Pu Wang ${ }^{5}{ }^{5}$ \\ ${ }^{1}$ The Affiliated Hospital of Southwest Medical University, Luzhou, Sichuan 646000, China \\ ${ }^{2}$ Department of Neurology, The 452nd Hospital of PLA, Chengdu, Sichuan, China \\ ${ }^{3}$ Key Laboratory for NeuroInformation of Ministry of Education, School of Life Science and Technology, University of Electronic \\ Science and Technology of China, Chengdu, Sichuan, China \\ ${ }^{4}$ Department of Neurology, Sichuan Provincial People's Hospital, 32 West Second Section First Ring Road, Chengdu, \\ Sichuan 610072, China \\ ${ }^{5}$ Department of Neurology, Chongzhou People's Hospital, Chongzhou, Sichuan, China
}

Correspondence should be addressed to Fuqiang Guo; guofuqiang2005@126.com and Pu Wang; wangpu1979@yeah.net

Received 3 October 2017; Revised 16 December 2017; Accepted 25 December 2017; Published 26 February 2018

Academic Editor: Jingqi Yan

Copyright (C) 2018 Chengqing Zhong et al. This is an open access article distributed under the Creative Commons Attribution License, which permits unrestricted use, distribution, and reproduction in any medium, provided the original work is properly cited.

\begin{abstract}
The aim of this study was to investigate the structural and functional connectivity (FC) of juvenile myoclonic epilepsy (JME) using resting state functional magnetic resonance imaging (rs-fMRI). High-resolution T1-weighted magnetic resonance imaging (MRI) and rs-fMRI data were collected in 25 patients with JME and in 24 control subjects. A FC analysis was subsequently performed, with seeding at the regions that demonstrated between-group differences in gray matter volume (GMV). Then, the observed structural and FCs were associated with the clinical manifestations. The decreased GMV regions were found in the bilateral anterior cerebellum, the right orbital superior frontal gyrus, the left middle temporal gyrus, the left putamen, the right hippocampus, the bilateral caudate, and the right thalamus. The changed FCs were mainly observed in the motor-related areas and the cognitiverelated areas. The significant findings of this study revealed an important role for the cerebellum in motor control and cognitive regulation in JME patients, which also have an effect on the activity of the occipital lobe. In addition, the changed FCs were related to the clinical features of JME patients. The current observations may contribute to the understanding of the pathogenesis of JME.
\end{abstract}

\section{Introduction}

Juvenile myoclonic epilepsy (JME) is characterized by transitory, bilateral symmetry, synchronous, and unrhythmic muscle jerks, generally, with no loss of consciousness [1]. The structural brain abnormalities are not found by conventional MRI and computed tomography in JME patients [1], but the development of hypersensitive neuroimaging technology illustrates the microstructural and functional changes of in JME patients [1]. Many alterations of subcortical structures have been reported in JME patients. Using proton magnetic resonance spectroscopy, the $\mathrm{N}$-acetyl aspartate (NAA) levels of the thalamus were reduced in JME patients, which indicated thalamus dysfunction is part of epileptogenesis in JME [2]. In ${ }^{1} \mathrm{H}$-magnetic resonance spectroscopy $\left({ }^{1} \mathrm{H}-\right.$ MRS) studies, significantly reduced concentrations of NAA in the prefrontal were observed, which demonstrated the abnormality of the prefrontal cortex in JME patients compared with controls $[3,4]$. When a VBM analysis was performed in JME patients, different results were observed in recent years. For instance, some studies found reduced GMVs in the prefrontal lobe $[5,6]$, the bilateral thalamus [7-11], supplementary motor area (SMA), the posterior cingulate gyrus [12], the insula, and bilateral cerebellar hemisphere [8]. Increased GMVs were found in the precentral gyrus, the parietal lobe, and the right middle temporal gyrus 
[13], and some studies even found increased GMVs in the frontal lobe. Of note, a recent study showed that the cerebellum had a decreased fractional anisotropy (FA) value in idiopathic generalized epilepsy, which demonstrated the microstructural integrity was changed [14]. At present, most of the VBM studies focused on the changes in the frontal lobe and thalamus; however, an increasing number of abnormal structural changes have been found in other brain regions.

To further understand the pathogenesis of JME, the resting state $\mathrm{FC}$ has proven to be an effective means for investigating the characteristics of brain activity, and it has been widely used to study epilepsy in recent years [15-17]. Vulliemoz et al. and Vollmar et al. demonstrated increased FC between the motor cortex and SMA based on cognitive task analysis in JME patients $[18,19]$, which hinted that cognitive activity triggered the myoclonic jerks [20]. Electrophysiological and functional neuroimaging studies suggested a functional disorder in the fronto-thalamo-frontal circuitry $[2,21]$ in JME patients. Synchronous electroencephalography and functional magnetic resonance imaging (EEG-fMRI) studies have demonstrated the generalized spike and wave discharge-related thalamic and default mode network (DMN) were deactivated in idiopathic generalized epilepsy (IGE); meanwhile, clear activation in the cerebellum has also been recorded during epileptic discharges [22]. Moreover, a reduced regional homogeneity ( $\mathrm{ReHo}$ ) value in the cerebellum was found in our previous study [23] using the rs-fMRI analysis in JME patients, which suggested the underlying spontaneous activity of the cerebellum is partially related to the pathogenesis of JME. Previous studies considered that the interactions between cortical and subcortical structures are important for the generation of generalized epilepsy [24, 25]. Therefore, epilepsy is regarded as a disruption of functional networks [26].

Previous studies have found the thalamo-frontal network plays an important role in the pathogenesis of JME. Although there have been many studies on the pathogenesis of JME, it is not fully elucidated. We therefore combined VBM and FC analysis using high-resolution T1 and rs-fMRI data to provide more evidence for understanding the potential pathophysiological mechanisms of JME.

\section{Methods}

2.1. Participants. Twenty-five patients (mean age: $24.68 \pm 6.4$ years, mean years of duration: $12.16 \pm 7.4$, and mean age of seizure onset: $12.16 \pm 3.9$ years, 17 females) with JME were recruited in the Center for Information in Medicine, University of Electronic Science and Technology of China. The diagnosis of JME was in line with clinical histories and electroencephalographic findings and was consistent with the International League Against Epilepsy (ILAE) classifications [1] (C. Zhong, P. Wang, F. Guo). All the conventional neuroimaging examinations, including CT and MRI scanning, displayed no structural abnormalities by visual inspection. Twenty-four healthy subjects were recruited as the sex- and age-matched control group (mean age: $25.41 \pm$ 7.7 years; 15 females). None of the controls had neurological or psychiatric disorders. This study was approved by the ethical committee of the University of Science and Technology of China according to the standards of the Declaration of Helsinki. Every patient provided signed informed consent.

2.2. Data Acquisition. All brain MR data was collected by a 3T GE MRI scanner with an eight-channel-phased array head coil (MR750; GE Discovery, Milwaukee, WI) at the MRI research center of the University of Electronic Science and Technology of China. The T1-weighted images were acquired using a three-dimensional fast spoiled gradient echo (T1-3DFSPGR) sequence $(\mathrm{TR}=6.008 \mathrm{msec}, \mathrm{TE}=1.984 \mathrm{msec}$, $\mathrm{FA}=90^{\circ}$, matrix $=256 \times 256, \mathrm{FOV}=25.6 \times 25.6 \mathrm{~cm}^{2}$, and slice thickness (no gap) $=1 \mathrm{~mm}$ ). Rs-fMRI data were acquired using gradient echo EPI sequences $(\mathrm{TR}=2000 \mathrm{~ms}, \mathrm{TE}=$ $30 \mathrm{~ms}, \mathrm{FA}=90^{\circ}$, matrix $=64 \times 64, \mathrm{FOV}=24 \times 24 \mathrm{~cm}^{2}$, slice thickness $=4 \mathrm{~mm}$ (no gap), and 35 slices per volume). All subjects underwent a $510 \mathrm{~s}$ resting state scan yielding 255 volumes. Subjects were asked to close their eyes and not sleep during the data scanning [27].

2.3. Voxel-Based Morphometry Analysis. The analysis of voxel-based morphometry was performed using the SPM8 package, which was implemented in MATLAB R2014 (available from http://dbm.neruo.uni-jena.de/vbm/). First, the obtained DICOM data was transferred into a nifit file, then the artifacts in all pictures were checked, and the images were repositioned to verify the origin of coordinates on the joint of anterior commissure.

Second, a diffeomorphic nonlinear registration algorithm (DARTEL, diffeomorphic anatomical registration through exponentiated lie algebra) was used to deform the GM partitions into a new customized reference space representing an average of all the subjects [28]. All T1 images were manually coregistered with the standard $\mathrm{T} 1$ template provided by SPM8. The coregistered images were then divided into GM, white matter (WM), and cerebrospinal fluid (CSF) using the unified segmentation procedure [29]. The resulting average size images were ulterior spatially normalized to Montreal Neurological Institute (MNI) space.

Third, the volume deformation caused by the nonlinear change was corrected with the Jacobian determents, and an isotropic Gaussian kernel ( $8 \mathrm{~mm}$ full width at half maximum) was used to smooth the modulated GM images.

Finally, the GMVs of the JME patient were compared with the normal control group using two sample $t$-tests in SPM8. The age and gender were corrected as confounding covariates. The level of significance for group differences was set at $p<0.05$ (FDR corrected). The identification of regions of interest (ROI) was based on the comparison of GMV.

2.4. Functional Connectivity Analysis. RS-fMRI data were preprocessed using the SPM8 software package (http://dbm. neruo.uni-jena.de/vbm/). The functional data were corrected for slice timing and head motion. To avoid the influence of magnetic field instability, the first five volumes were removed. Subjects with head motion more than $3 \mathrm{~mm}$ (translation) and $1.5^{\circ}$ (rotation) during scanning were excluded. 
TABLe 1: Demographic data of 25 juvenile myoclonic epilepsy patients.

\begin{tabular}{|c|c|c|c|c|c|}
\hline Number & Gender & Age (years) & Age at seizure onset (year) & Antiepileptic drugs & History/family history \\
\hline 1 & $\mathrm{~F}$ & 17 & 13 & VPA & Sister with JME \\
\hline 2 & $\mathrm{~F}$ & 17 & 13 & VPA & Sister with JME \\
\hline 3 & M & 26 & 8 & MVP & None \\
\hline 4 & M & 21 & 17 & LEV & Grandmother with epilepsy \\
\hline 5 & $\mathrm{~F}$ & 20 & 6 & VPA, LTG & None \\
\hline 6 & $\mathrm{~F}$ & 34 & 14 & - & None \\
\hline 7 & $\mathrm{~F}$ & 27 & 16 & - & Daughter with epilepsy \\
\hline 8 & $\mathrm{~F}$ & 16 & 13 & LTG & None \\
\hline 9 & M & 30 & 9 & CBZ, LEV & None \\
\hline 10 & M & 34 & 14 & VPA, VPM & None \\
\hline 11 & $\mathrm{~F}$ & 25 & 10 & VPA & None \\
\hline 12 & $\mathrm{~F}$ & 22 & 14 & VPA & None \\
\hline 13 & $\mathrm{~F}$ & 24 & 18 & CBZ, VPA & None \\
\hline 14 & M & 37 & 12 & VPA & None \\
\hline 15 & $\mathrm{~F}$ & 23 & 7 & VPA & None \\
\hline 16 & $\mathrm{~F}$ & 17 & 10 & VPA & None \\
\hline 17 & $\mathrm{~F}$ & 29 & 10 & VPA, VPM & None \\
\hline 18 & $\mathrm{~F}$ & 33 & 20 & VPM, LTG & None \\
\hline 19 & M & 18 & 14 & VPM & None \\
\hline 20 & $\mathrm{~F}$ & 25 & 21 & VPM & None \\
\hline 21 & M & 22 & 15 & VPA & None \\
\hline 22 & M & 38 & 8 & VPA & Brother with epilepsy \\
\hline 23 & M & 22 & 8 & VPM & None \\
\hline 24 & $\mathrm{~F}$ & 21 & 11 & VPM & None \\
\hline 25 & $\mathrm{~F}$ & 19 & 12 & MVP & Uncle with IGE \\
\hline
\end{tabular}

M: male; F: female; VPA: valproic acid; VPM: valpromide; MVP: magnesium valproate; LTG: lamotrigine; TOP: topiramate; CBZ: carbamazepine.

Next, the corrected imaging data were spatially normalized to standard MNI space and resampled to $3 \times 3 \times 3 \mathrm{~mm}^{3}$. Then, spatial smoothing was applied with an $8 \mathrm{~mm}$ full-width at half maximum (FWHM) Gaussian kernel and bandpass filter $(0.01-0.08 \mathrm{~Hz})$ that dealt with the timing sequences. Finally, head motion parameters, CSF and WM, and globe signals were regressed out in the general linear model for each subject. After preprocessing of rs-fMRI data, the seeds were defined with a radius of $3 \mathrm{~mm}$ and a center voxel at the peak of the between-group difference in VBM analysis. For a given seed, the functional connectivity was calculated between the time courses from each seed and voxel in the whole brain using REST software (http://www.restfmri.net). For each group, an individual functional connectivity map was analyzed with a random effect one-sample $t$-test to identify voxels that showed a significant correlation with the seed. The two-sample $t$-test was used to compare the functional connections between groups. The group comparison was restricted to an explicit mask from the union set of the significant one-sample $t$-test results. Furthermore, a correlation analysis was performed to detect the relation between the FC that showed a significant between-group difference and the clinical features, including age of seizure onset and duration of epilepsy, with controlling effects of gender and age [27].

\section{Results}

3.1. Demographic and Clinical Characteristics. There were no significant differences in age or sex between the patients and controls. The detailed demographic information of patients is shown in Table 1. No one was excluded because of excessive head motion.

3.2. Voxel-Based Morphometric Analysis. Compared with the control group, patients with JME showed significantly decreased GM volumes of regions in the cerebellum (bilateral anterior of cerebellum and vermis), the right thalamus, bilateral caudate, part of the cerebral cortex (the orbital superior frontal gyrus and left middle temporal gyrus), the right hippocampus, and the left putamen (Table $2, p<0.05$, FDR corrected) (Figure 1). We used the xjView toolbox (http://www. alivelearn.net/xjview) to visualize the results. No brain regions showed increased gray matter volume in patients with JME. Thus, based on the results from VBM, we chose ten ROIs for the following functional connectivity analysis.

3.3. Functional Connectivity Analysis. Resting state FC analysis revealed the altered functional connections with selected ROIs $(p<0.05$, FDR corrected; Figure 2$)$. For the seed at the right anterior cerebellum (MNI coordinate: $x=34, y=$ 
TABLE 2: The cluster with decreased GM volumes in patients with JME compared with controls.

\begin{tabular}{|c|c|c|c|c|c|c|c|}
\hline \multirow{2}{*}{ Regions } & \multirow{2}{*}{ Abbreviation } & \multirow{2}{*}{$\mathrm{L} / \mathrm{R}$} & \multicolumn{3}{|c|}{ MNI coordinates } & \multirow{2}{*}{ Peak $T$ value } & \multirow{2}{*}{ Cluster size } \\
\hline & & & $x$ & $y$ & $z$ & & \\
\hline Cerebellum_crus1 & CERE_CRUS 1 & $\mathrm{R}$ & 34 & -84 & -31 & -8.20 & 79 \\
\hline Cerebellum_crus1 & CERE_CRUS 1 & $\mathrm{~L}$ & -26 & -71 & -29 & -4.46 & - \\
\hline Frontal_sup_Orb & ORBsup & $\mathrm{R}$ & 10 & 64 & -13 & -6.50 & 82 \\
\hline Temporal_Mid & MTG & $\mathrm{L}$ & -67 & -33 & -4 & -7.57 & 78 \\
\hline Putamen & PUT & $\mathrm{L}$ & -18 & 1 & -10 & -6.03 & - \\
\hline Hippocampus & HIPP & $\mathrm{R}$ & 30 & -8 & 16 & -4.80 & - \\
\hline Caudate & CAU & $\mathrm{L}$ & -10 & 12 & -5 & -5.84 & 354 \\
\hline Caudate & CAU & $\mathrm{R}$ & 12 & 21 & 3 & -5.65 & - \\
\hline Vemis_4_5 & $\mathrm{CBV}$ & $\mathrm{R}$ & 6 & -55 & -22 & -5.00 & 124 \\
\hline Thalami & $\mathrm{TH}$ & $\mathrm{R}$ & 18 & -23 & 13 & -5.03 & 28 \\
\hline
\end{tabular}

L: left; R: right.

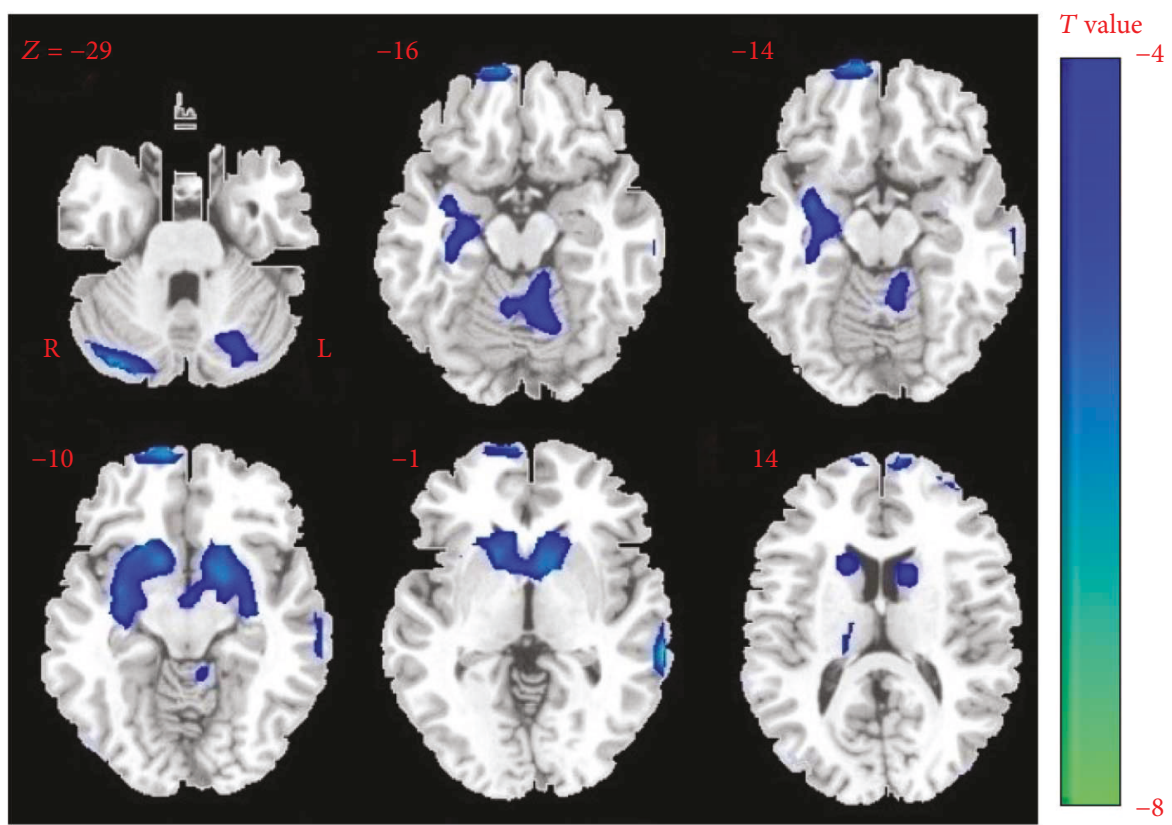

FIGURE 1: The difference of GM volume between groups. The decreased volume in patients contrasting to healthy controls is shown in cool color. No GM volume increase was observed in patients.

-84 , and $z=-31$ ), patients showed significantly increased FCs with the left posterior cerebellum, right middle occipital gyrus, left superior occipital gyrus, and bilateral postcentral gyrus, and decreased FCs with the left superior frontal gyrus, bilateral orbital inferior frontal gyrus, left middle temporal gyrus, and the left SMA. For the left anterior cerebellum (MNI coordinate: $x=-26, y=-71$, and $z=-29$ ), increased FCs were found with the cluster of the left middle occipital gyrus, the cluster of the right superior occipital gyrus, and the bilateral anterior cerebellum. Decreased FCs to the left anterior cerebellum were also found in the cluster of the right middle frontal gyrus, the right SMA, the left orbital inferior frontal gyrus, and the left middle frontal lobe. For the seed at the vermis (MNI coordinate: $x=6, y=-55$, and $z=-22$ ), patients showed increased functional connections with the left middle temporal gyrus, the left fusiform gyrus, and the cluster of the right precuneus lobule and decreased FCs with the left SMA, the clusters of the bilateral triangular inferior frontal gyrus.

For the left putamen (MNI coordinate: $x=-18, y=1, z=$ -10), patients showed increased FCs with the right hippocampus and the left middle cingulate gyrus and decreased FCs with the bilateral triangular inferior frontal gyrus, the right precentral gyrus, the bilateral superior parietal lobule, and the left inferior parietal lobe. For the left caudate (MNI coordinate: $x=-10, y=12$, and $z=-5$ ), patients showed increased FCs with the right inferior cerebellum and the left supramarginal gyrus and decreased FCs with the left orbital superior frontal lobe and the left paracentral lobule. For the right caudate (MNI coordinate: $x=12, y=21$, and $z=3$ ), patients showed increased functional connections with the bilateral posterior cerebellum and the bilateral caudate. Decreased functional connections to the right caudate were also found in the right orbital superior frontal gyrus and 


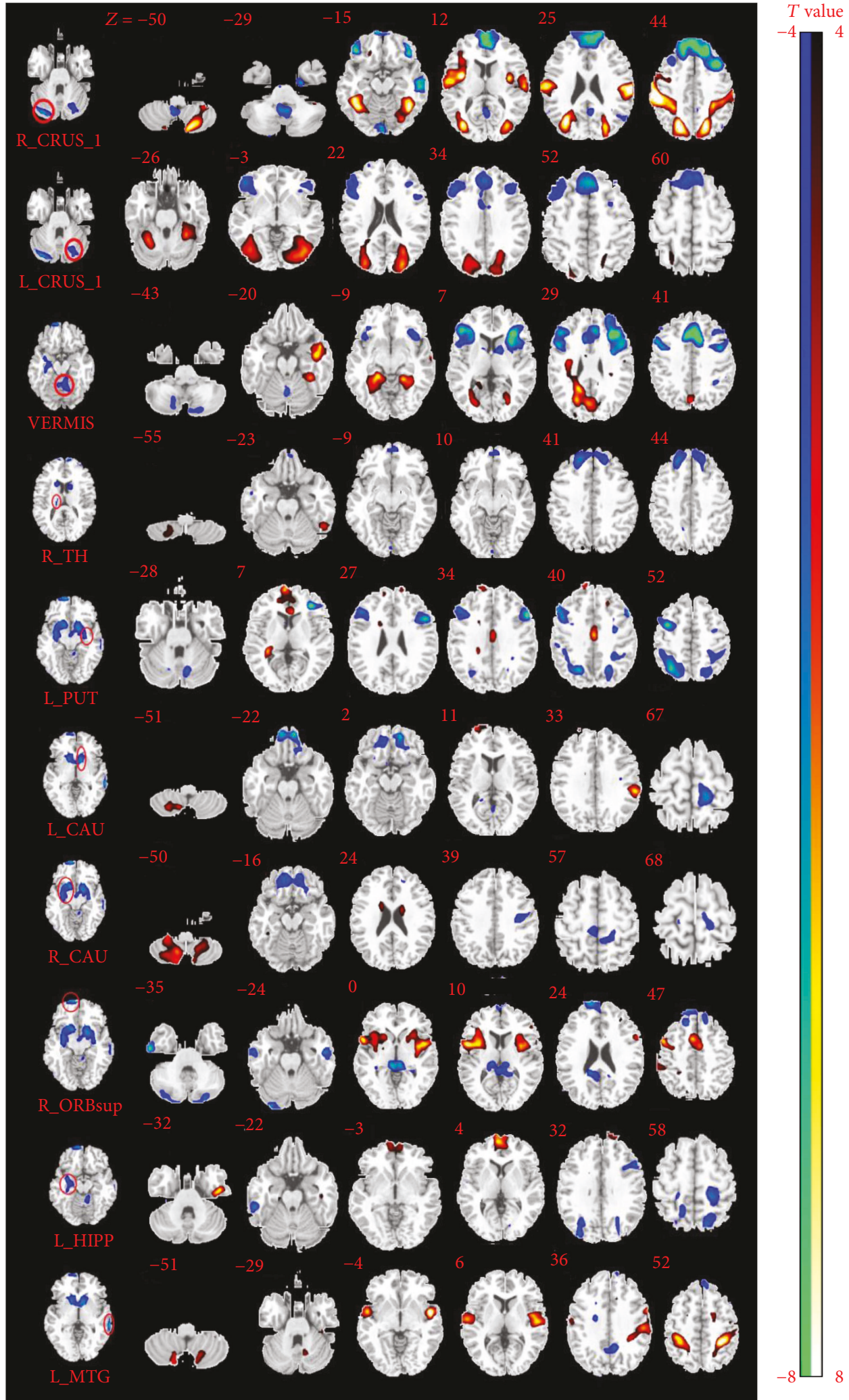

FIgURE 2: The different functional connection (FC) between groups. The position of seeds in AAL regions is shown on the left side of the figure. Each row shows changed FCs with left seed. The hot color means increased FCs, and cool color represents decreased FCS with the seeds. 
the left postcentral gyrus. For the seed at the right thalamus (MNI coordinate: $x=18, y=-23$, and $z=13$ ), patients showed increased functional connections with the right posterior cerebellum and the bilateral thalamus and decreased FCs with the right superior frontal gyrus and left medial superior frontal gyrus.

For the seed at the right orbital superior frontal gyrus (MNI coordinate: $x=10, y=64$, and $z=-13$ ), patients showed increased FCs with the bilateral insula gyrus and the bilateral middle cingulate gyrus and decreased FCs to the right anterior cerebellum, the right inferior temporal gyrus, the left middle temporal gyrus, vermis, and the right medial superior frontal gyrus.

For the seed at the left middle temporal gyrus (MNI coordinate: $x=-67, y=-33$, and $z=-44$ ), patients showed increased FCs with the clusters of the bilateral superior temporal gyrus, clusters of the left inferior parietal lobule, and clusters of the right postcentral gyrus. FCs were decreased to the left middle temporal gyrus with the right anterior cerebellum, the left precuneus lobule, and the left medial superior frontal lobe. For the right hippocampus (MNI coordinate: $x=30, y=-8$, and $z=16$ ), patients showed increased FCs with the left inferior temporal gyrus and clusters of the bilateral medial superior frontal gyrus and decreased FCs with the right inferior temporal gyrus, the left postcentral gyrus, the right Rolandic operculum, and the bilateral occipito-parietal lobules. These differences in functional connections are fully visualized in Table 3.

3.4. The Correlation Analysis between Functional Connections and Clinical Features, Including Onset Age and Duration of Epilepsy. The FC in the brain regions with significant between-group differences were partially related to the duration and onset age in JME patients, while controlling for age, gender, and head motions. First, there were no significant relationships between the VBM and either the duration or age of onset in JME patients.

For functional connectivity, significant positive correlations with onset age were found in the FCs between the right caudate and the right posterior cerebellum (MNI coordinate: $x=-14, y=-68$, and $z=-45)(r=0.46, P=0.02$, Figure $3(\mathrm{a}))$, but there was a negative correlation in FCs between the left putamen and the right occipital superior gyrus $(r=-0.04$, $P=0.04$, Figure 3(b)). In addition, FCs between the left superior frontal gyrus and the right superior frontal gyrus $(r=-0.46, P=0.02$, Figure 3(c)) and the FCs between the right hippocampus and the right orbital inferior frontal gyrus $(r=-0.56, P=0.003$, Figure $3(d))$ showed negative correlations with the illness duration of JME.

\section{Discussion}

In the present study, we investigated alterations of the brain structure and functional connections in JME patients. Compared with the control group, the main findings in JME patients were as follows: (1) The decreased GMVs were mainly located at the cerebellum, the basal ganglia, the thalamus, and parts of the cerebral cortex including the frontal area, the parieto-occipital lobe, the left temporal lobe, and the memory-related hippocampal gyrus. (2) In the functional connectivity analysis, we found significantly increased FCs in the motor-related and sensory-related areas, decreased FCs in the cognitive-related areas, and changed FCs in the language- and memory-related regions. (3) We also found significant differences in FCs in the brain regions that were correlated with onset age and duration of epilepsy in JME patients.

4.1. Alterations in Motor-Related Regions. Previous studies suggested that the anterior cerebellum participated in feedforward motor control, and the posterior cerebellum participated in transforming visual information feedback into precise motor adjustments [30]. Researchers have demonstrated that the cerebellum receives a large amount of information widely from the cerebral cortex [31, 32], then its efferents, with GABAergic inhibition via the thalamus, project to the primary motor cortex, and the premotor cortex participates in movement regulation [33], while the efferents project to prefrontal and posterior parietal areas participate in cognition and visuospatial function $[34,35]$. In epilepsy, some studies have demonstrated structural and functional alterations in the cerebellum. For example, reduced fractional anisotropy values were detected in the right cerebellum, and reduced FCs between the right cerebellar and the left middle frontal gyrus were also detected $[14,36]$. In the current study, a decreased GMV of the bilateral anterior cerebellum, increased FCs between the posterior cerebellum and the right thalamus, and even increased FCs between the anterior cerebellum and the posterior cerebellum were found, which might be related to the function of feedforward motor control [37]. These findings may indicate that the increased activity of the cerebellum, accompanied by the enhanced abnormal movement in JME patients, reflected the feedback mechanism in movement regulation. In addition, the findings showed disruption of the cerebello-thalamo-cortical circuits in JME patients. We also observed decreased FCs between the cerebellum and SMA. The SMA usually suggests involvement in the postural stabilization of the body, coordination, and movement $[38,39]$. Thus, we presumed that the decreased FCs may testify declined activation of the coordination area.

For the motor-related basal ganglia regions, the left putamen and the bilateral caudate showed decreased GMV and increased FCs between the bilateral caudate and the posterior cerebellum. The basal ganglia are widely considered to play an important role in the regulation of discharges [40]. The caudate is especially involved in interictal spike-wave discharges and generalized seizure activity, which was found by $\mathrm{Li}$ et al. [41]. In addition, our findings of increased FCs between the caudate and the posterior cerebellum revealed a modulation effect on the activity of epilepsy, because of the feedback effect of the posterior cerebellum in motor regulation mechanisms. These findings are in line with our previous study of the basal ganglia network in idiopathic generalized epilepsy (2012) [42]. In addition, we observed the FCs between the right caudate and the right posterior cerebellum were positively related to the onset age in JME patients. 
TABLE 3: Altered intrinsic functional connectivity seeded at regions with decreased GM volume in patients with JME compared with controls. CRUS 1: cerebellum crus 1; ORBsup: superior orbital frontal gyrus; MTG: middle temporal gyrus; PUT: putamen; HIPP: hippocampus; CAU: caudate; CBV: cerebellum vermis; TH: thalamus. L: left; R: right.

\begin{tabular}{|c|c|c|c|c|c|c|}
\hline \multirow{2}{*}{ ROI } & \multirow{2}{*}{ Brain region } & \multicolumn{3}{|c|}{ MNI coordinates } & \multirow{2}{*}{$T$ value } & \multirow{2}{*}{ Voxel number } \\
\hline & & $x$ & $y$ & $z$ & & \\
\hline \multirow[t]{11}{*}{ R_CRUS_1 } & Occipital_Mid_R & 27 & -65 & 27 & 6.33 & 1027 \\
\hline & Occipital_Sup_L & -23 & -69 & 36 & 4.84 & 777 \\
\hline & Cerebellum_8_L & -25 & -69 & -52 & 4.87 & 120 \\
\hline & Postcentral_R & 58 & -15 & 26 & 4.79 & \\
\hline & Parietal_Sup_R & 18 & -70 & 47 & 5.46 & \\
\hline & Postcentral_L & -33 & -36 & 47 & 4.36 & \\
\hline & Frontal_Sup_L & -19 & 21 & 54 & -3.68 & 1054 \\
\hline & Temporal_Mid_L & -56 & -29 & -9 & -4.94 & 271 \\
\hline & Frontal_Inf_Orb_L & -41 & 34 & -18 & -4.72 & 130 \\
\hline & Frontal_Inf_Orb_R & 42 & 36 & -18 & -5.26 & 72 \\
\hline & Supp_Motor_Area_L & -8 & 21 & 64 & -6.44 & \\
\hline \multirow[t]{8}{*}{ L_CRUS_1 } & Occipital_Mid_L & -27 & -85 & 22 & 5.84 & 699 \\
\hline & Occipital_Sup_R & 28 & -83 & 22 & 5.44 & 497 \\
\hline & Cerebellum_6_R & 28 & -53 & -26 & 5.93 & \\
\hline & Cerebellum_6_L & -32 & -44 & -26 & 6.29 & \\
\hline & Frontal_Mid_R & 46 & 26 & 39 & -4.45 & 744 \\
\hline & Frontal_Mid_L & -36 & 28 & 39 & -4.14 & 175 \\
\hline & Frontal_Inf_Orb_L & -48 & 36 & -7 & -3.81 & 114 \\
\hline & Supp_Motor_Area_R & 7 & 22 & 61 & -4.38 & \\
\hline \multirow[t]{6}{*}{ CBV } & Temporal_Mid_L & -48 & 2 & -21 & 3.67 & 96 \\
\hline & Fusiform_L & -36 & -45 & -18 & 4.85 & 148 \\
\hline & Precuneus_R & 18 & -52 & 24 & 4.83 & 232 \\
\hline & Supp_Motor_Area_L & -5 & 18 & 45 & -6.46 & 525 \\
\hline & Frontal_Inf_Tri_L & -36 & 30 & 0 & -6.18 & 411 \\
\hline & Frontal_Inf_Tri_R & 48 & 33 & 6 & -4.88 & 336 \\
\hline \multirow[t]{5}{*}{ R_TH } & Cerebellum_8_R & 18 & -51 & -60 & 3.92 & 50 \\
\hline & Thalamus_R & 18 & -15 & 12 & 3.81 & 25 \\
\hline & Thalamus_L & -15 & -15 & 9 & 3.68 & 28 \\
\hline & Frontal_Sup_R & 15 & 39 & 39 & -5.63 & 174 \\
\hline & Frontal_Sup_Medial_L & -10 & 61 & 16 & -3.93 & 149 \\
\hline \multirow[t]{6}{*}{ R_CAU } & Cerebellum_8_R & 12 & -60 & -54 & 5.95 & 346 \\
\hline & Cerebellum_8_L & -14 & -68 & -45 & 4.50 & 171 \\
\hline & Caudate_L & -15 & -3 & 21 & 4.39 & 35 \\
\hline & Caudate_R & 15 & 3 & 21 & 4.23 & 30 \\
\hline & Frontal_Sup_Orb_R & 12 & 52 & -22 & -5.04 & 168 \\
\hline & Postcentral_L & -40 & -16 & 36 & -4.22 & 100 \\
\hline \multirow[t]{4}{*}{ L_CAU } & Cerebellum_8_R & 12 & -66 & -51 & 3.69 & 54 \\
\hline & SupraMarginal_L & -57 & -33 & 30 & 4.77 & 107 \\
\hline & Paracentral_Lobule_L & -13 & -26 & 78 & -4.50 & 74 \\
\hline & Frontal_Sup_Orb_L & -13 & 44 & -24 & -3.48 & 68 \\
\hline \multirow[t]{6}{*}{ L_PUT } & Cingulum_Mid_L & -3 & -15 & 36 & 4.38 & 55 \\
\hline & Hippocampus_R & 36 & -30 & -6 & 4.80 & 32 \\
\hline & Frontal_Inf_Tri_L & -36 & 39 & 3 & -5.78 & 65 \\
\hline & Precentral_R & 36 & 1 & 51 & -5.17 & 83 \\
\hline & Frontal_Inf_Tri_R & -53 & 19 & 29 & -5.21 & 86 \\
\hline & Parietal_Sup_L & -24 & -68 & 43 & -3.46 & 122 \\
\hline
\end{tabular}


TABLE 3: Continued.

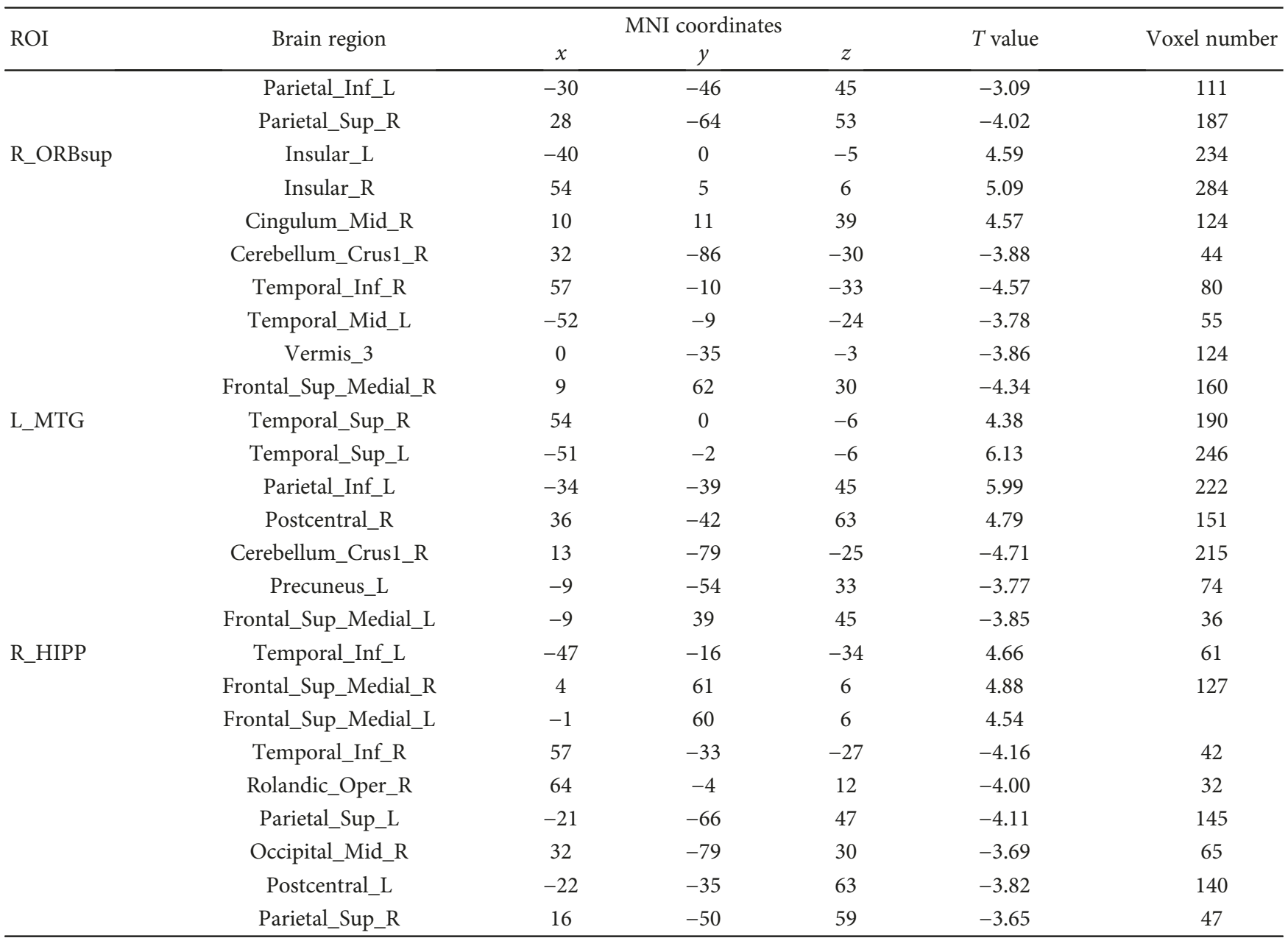

Furthermore, significantly increased FCs between the anterior cerebellum and the occipital lobe were observed in the JME patients. The occipital lobe mainly manifested as photosensitive property in epilepsy patients, especially in idiopathic occipital lobe epilepsies [43]. An existing study showed that $30 \%$ of JME patients are photosensitive [44]. However, there were no definite photosensitive seizures in all patients recruited in the current study. This phenomenon may be understood by the increased FCs with the cerebellum, which affected the function of the occipital cortex and inhibited the function of the cerebellum. The FCs between the left putamen and the right occipital superior gyrus were negatively related to the onset age in JME patients, and this may further hint to photosensitivity seizure reduction following the increased FCs with the cerebellum.

4.2. Alterations in Cognition-Related Regions. The efferents of the cerebellum project to prefrontal areas and posterior parietal areas that participate in cognition and visuospatial functions $[45,46]$. There is hard evidence that has suggested the function of the frontal lobe mainly manifests as working memory [47], executive functions [48], and prospective memory [49]. In our study, the reduced GMV of the right orbital superior frontal gyrus and decreased FCs between anterior cerebellum and frontal lobe in JME patients might imply the cognitive activity declination. This phenomenon may be caused by the preponderant discharges of the frontal lobe [50] in JME, which lead to the decreased GMV of local neuron or neuron cell impairment [51, 52]. Meanwhile, the decreased FCs between the bilateral superior frontal gyrus in patients is negatively related with the illness duration (Figure 3(c)). This finding may demonstrate that the cognitive activity gradually aggravated by the extension of disease duration. Besides, we also found the right anterior cerebellum and the bilateral intraparietal sulcus (IPS) showed significantly increased FCs (showed in Figure 2), and the IPS is known as important nodes of the dorsal attention network (DAN) [53], which influence the visual area when attended to spatial orienting [54]. In addition, the increased FCs for anterior cerebellum and vermis to the precuneus has been founded, and the latter also involves in visuospatial processing [55]; these findings may further suggest the increased activity of cerebellum in the regulation of visuospatial plan in JME patients.

Another remarkable finding in our study is the decreased GMV of the right superior orbital frontal gyrus and increased FC between the right superior orbital frontal gyrus and 

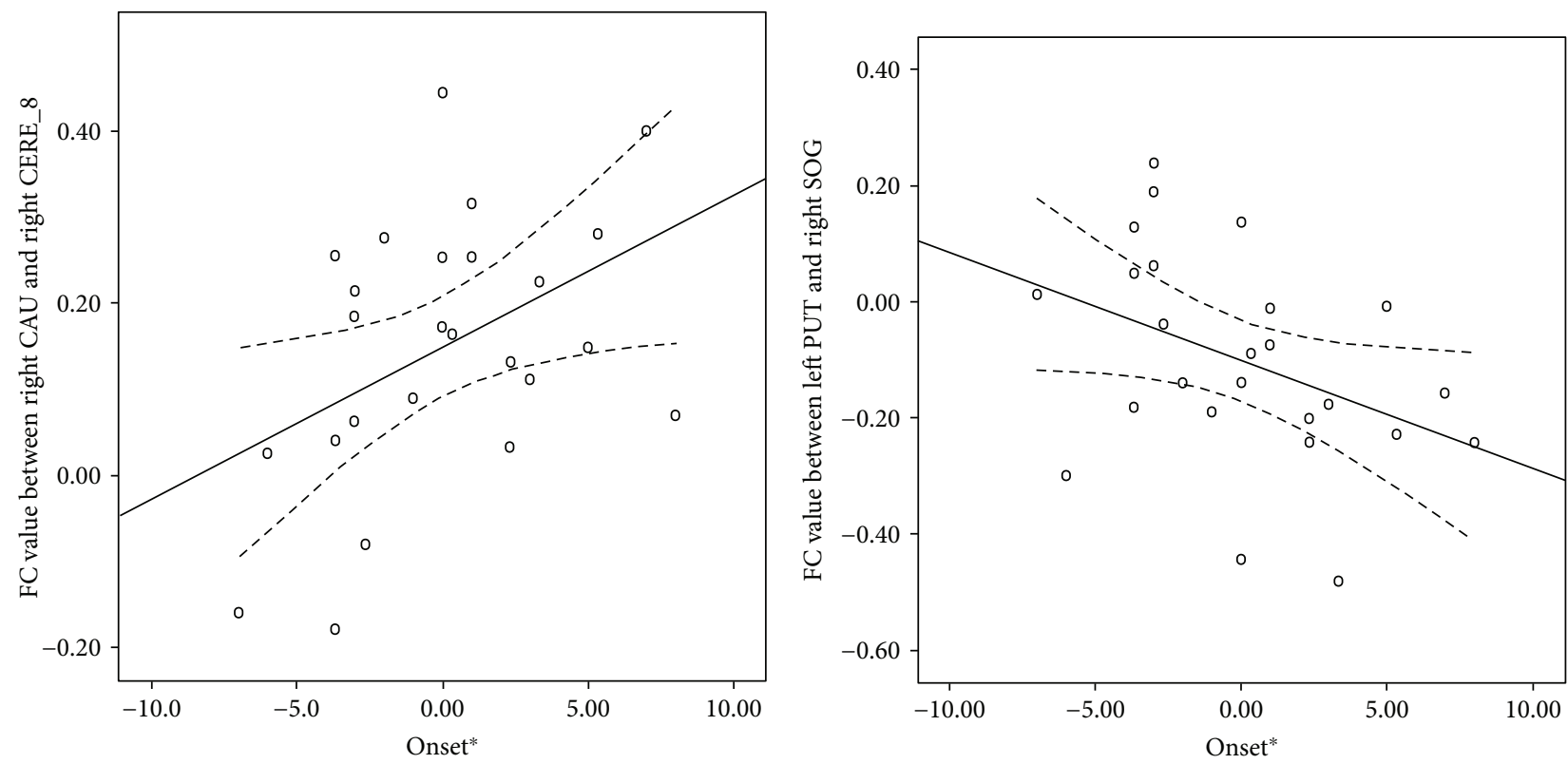

$r=0.46$

$p=0.02$

$$
\begin{aligned}
& r=-0.40 \\
& p=0.04
\end{aligned}
$$

(a)

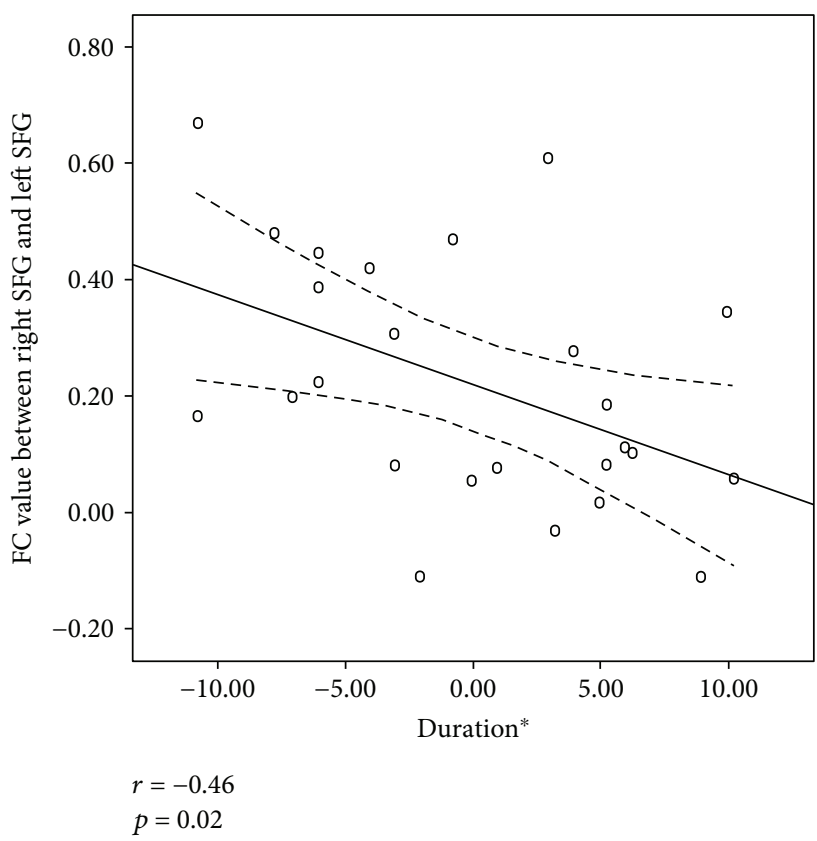

(c)

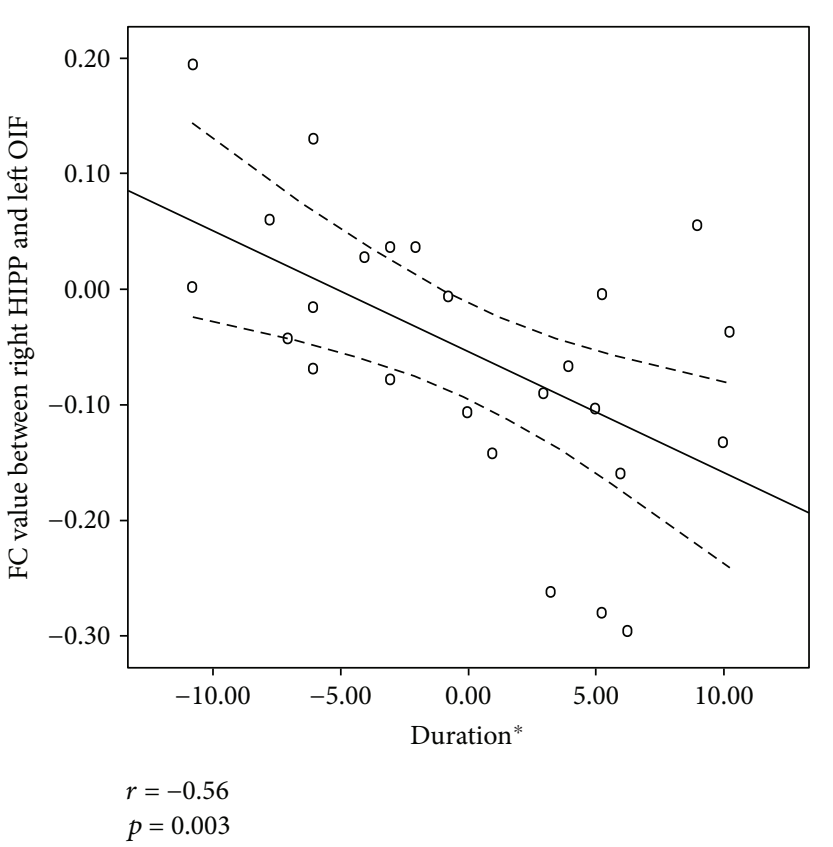

(d)

FIgURE 3: The correlation between FCs and clinical features. The FC between the right caudate and right cerebellum crus 8 showed positive correlations with the onset of age in JME patients $(r=0.46, p=0.02)$. (a) FC between left putamen and right occipital superior gyrus showed negative correlations with the onset of age $(r=-0.04, p=0.04)$. (b) FCs between left superior frontal gyrus and right superior frontal gyrus showed negative correlations with the illness duration $(r=-0.46, p=0.02)$. (c) And FCs between right hippocampus and right orbital inferior frontal gyrus $(r=-0.56, p=0.003)(\mathrm{d})$ also showed negative correlations with the illness duration of JME.

bilateral insula. The insula is usually involved in consciousness, emotion, and the body's homeostasis, which are the nodes of the salience network (SN) and play a crucial role in switching between the default mode network and central executive network [56]. This is consistent with the previous diffusion tensor imaging study, which revealed the insula interconnected to orbitofrontal cortex [57]. The increased FCs represent the increasing activity of insula and superior orbital frontal gyrus, which would inflect the enhanced interaction within limbic system in JME. Alternatively, it may owe to the more anxiety/arousal during MRI scanning [58] in patients than healthy controls. 
Taken together, these findings suggest that the regulation ability of cerebellum in cognition was decreased in JME patients, and decreased FCs between cognitive-related regions in JME patients were also found.

4.3. Alterations in Hippocampus and Temporal Lobule. Temporal lobe, especially mesial temporal structure, was affected by epilepsy activity in different types of epilepsy. In this study, the decreased GMV of the right hippocampus was observed. Consistent with our findings, the atrophy of hippocampus has already been described in JME patients in the previous study [5]. The potential reason included that the production of new neurons can be negatively affected by epileptic seizures [59], and the hyperexcitability can result in cytotoxicity and cell death [51].

Furthermore, we found decreased GMV in the left middle temporal gyrus which shows increased FC with left inferior parietal lobule, right postcentral gyrus, and left superior temporal gyrus. Evidence showed the left temporal lobe takes part in auditory and visual processing streams [60]. The postcentral gyrus is located at the primary somatosensory cortex, which is concerned with the main sense of touch. The function of these areas mainly involves in language, auditory processing, and sense of touch. Thus, we presume that the increased FC between these regions may reflect the compensation mechanism between functional similarity regions [61].

Notably, there are some shortcomings in our study. Firstly, the subjects are relatively small, so we cannot group it and discuss it further. If a group of patients with untreated epilepsy was involved in the control, there should be a more comprehensive explanation for the results. The sample size should gradually increase as our research continues. Secondly, if combined with EEG detect in the fMRI scanning, it would be better to access the correlation between epileptiform discharge and cognitive. In addition, the antiepileptic drugs may have some effect on the discharge of neuron and cognition; meanwhile, we also believe that comparing plus-treatment patients with epilepsy directly to a group of healthy patients can reflect the effects of epilepsy itself on the brain [62]. We would attempt to reduce these defects as far as possible in the future and improve the method. Finally, intelligence and mental test should be accomplished in the future work.

\section{Conclusion}

In summary, patients with JME showed focal structure abnormalities, especially the cerebellum, and found changed FCs in the motor-related area and cognitive-related regions. These findings revealed the important role of cerebellum in the pathogenesis of JME and may help us deeply understand the dysfunction in JME patients.

\section{Conflicts of Interest}

None of the authors has any conflict of interest to disclose.

\section{Acknowledgments}

This study was supported by the National Nature Science Foundation of China (nos. 81771822, 81271547).

\section{References}

[1] A. T. Berg, S. F. Berkovic, M. J. Brodie et al., "Revised terminology and concepts for organization of seizures and epilepsies: report of the ILAE commission on classification and terminology, 2005-2009," Epilepsia, vol. 51, no. 4, pp. 676-685, 2010.

[2] S. B. Mory, L. M. Li, C. A. M. Guerreiro, and F. Cendes, "Thalamic dysfunction in juvenile myoclonic epilepsy: a proton MRS study," Epilepsia, vol. 44, no. 11, pp. 1402-1405, 2003.

[3] R. J. Simister, M. A. McLean, G. J. Barker, and J. S. Duncan, "Proton MRS reveals frontal lobe metabolite abnormalities in idiopathic generalized epilepsy," Neurology, vol. 61, no. 7, pp. 897-902, 2003.

[4] I. Savic, Y. Osterman, and G. Helms, "MRS shows syndrome differentiated metabolite changes in human-generalized epilepsies," NeuroImage, vol. 21, no. 1, pp. 163-172, 2004.

[5] W. S. Tae, S. B. Hong, E. Y. Joo et al., "Structural brain abnormalities in juvenile myoclonic epilepsy patients: volumetry and voxel-based morphometry," Korean Journal of Radiology, vol. 7, no. 3, pp. 162-172, 2006.

[6] F. G. Woermann, S. L. Free, M. J. Koepp, S. M. Sisodiya, and J. S. Duncan, "Abnormal cerebral structure in juvenile myoclonic epilepsy demonstrated with voxel-based analysis of MRI," Brain, vol. 122, no. 11, pp. 2101-2108, 1999.

[7] J. H. Kim, J. K. Lee, S. B. Koh et al., "Regional grey matter abnormalities in juvenile myoclonic epilepsy: a voxel-based morphometry study," NeuroImage, vol. 37, no. 4, pp. 11321137, 2007.

[8] K. Lin, A. P. Jackowski, H. Carrete Júnior et al., "Voxel-based morphometry evaluation of patients with photosensitive juvenile myoclonic epilepsy," Epilepsy Research, vol. 86, no. 2-3, pp. 138-145, 2009.

[9] D. T. Pulsipher, M. Seidenberg, L. Guidotti et al., "Thalamofrontal circuitry and executive dysfunction in recent-onset juvenile myoclonic epilepsy," Epilepsia, vol. 50, no. 5, pp. 1210-1219, 2009.

[10] C. H. Chan, R. S. Briellmann, G. S. Pell, I. E. Scheffer, D. F. Abbott, and G. D. Jackson, "Thalamic atrophy in childhood absence epilepsy," Epilepsia, vol. 47, no. 2, pp. 399-405, 2006.

[11] C. Ciumas and I. Savic, "Structural changes in patients with primary generalized tonic and clonic seizures," Neurology, vol. 67, no. 4, pp. 683-686, 2006.

[12] J. O'Muircheartaigh, C. Vollmar, G. J. Barker et al., "Focal structural changes and cognitive dysfunction in juvenile myoclonic epilepsy," Neurology, vol. 76, no. 1, pp. 34-40, 2010.

[13] M. Liu, L. Concha, C. Beaulieu, and D. W. Gross, "Distinct white matter abnormalities in different idiopathic generalized epilepsy syndromes," Epilepsia, vol. 52, no. 12, pp. 22672275, 2011.

[14] Y. Li, H. Du, B. Xie et al., "Cerebellum abnormalities in idiopathic generalized epilepsy with generalized tonic-clonic seizures revealed by diffusion tensor imaging," PLoS One, vol. 5, no. 12, article e15219, 2010.

[15] D. Zhang, A. Z. Snyder, J. S. Shimony, M. D. Fox, and M. E. Raichle, "Noninvasive functional and structural connectivity 
mapping of the human thalamocortical system," Cerebral Cortex, vol. 20, no. 5, pp. 1187-1194, 2010.

[16] C. Luo, C. Qiu, Z. Guo et al., "Disrupted functional brain connectivity in partial epilepsy: a resting-state fMRI study," PLoS One, vol. 7, no. 1, article e28196, 2011.

[17] C. Luo, T. Yang, S. Tu et al., "Altered intrinsic functional connectivity of the salience network in childhood absence epilepsy," Journal of the Neurological Sciences, vol. 339, no. 1-2, pp. 189-195, 2014.

[18] S. Vulliemoz, C. Vollmar, M. J. Koepp et al., "Connectivity of the supplementary motor area in juvenile myoclonic epilepsy and frontal lobe epilepsy," Epilepsia, vol. 52, no. 3, pp. 507$514,2011$.

[19] C. Vollmar, J. O'Muircheartaigh, M. R. Symms et al., "Altered microstructural connectivity in juvenile myoclonic epilepsy: the missing link," Neurology, vol. 78, no. 20, pp. 1555-1559, 2012.

[20] Y. Inoue, M. Seino, H. Kubota, K. Yamakaku, M. Tanaka, and K. Yagi, "Epilepsy with praxis induced seizures," in Epileptic Seizures and Syndromes, P. Wolf, Ed., pp. 81-91, John Libbey, London, UK, 1994.

[21] F. Moeller, H. R. Siebner, S. Wolff et al., "Changes in activity of striato-thalamo-cortical network precede generalized spike wave discharges," NeuroImage, vol. 39, no. 4, pp. 1839-1849, 2008.

[22] J. Gotman, C. Grova, A. Bagshaw, E. Kobayashi, Y. Aghakhani, and F. Dubeau, "Generalized epileptic discharges show thalamocortical activation and suspension of the default state of the brain," Proceedings of the National Academy of Sciences of the United States of America, vol. 102, no. 42, pp. 1523615240, 2005.

[23] S. Jiang, C. Luo, Z. Liu et al., “Altered local spontaneous brain activity in juvenile myoclonic epilepsy: a preliminary restingstate fMRI study," Neural Plasticity, vol. 2016, Article ID 3547203, 7 pages, 2016.

[24] H. H. Jasper and J. Kershman, "Electroencephalographic classification of the epilepsies," Archives of Neurology And Psychiatry, vol. 45, no. 6, pp. 903-943, 1941.

[25] W. Penfield and T. C. Erickson, "Epilepsy and cerebral localization," The American Journal of the Medical Sciences, vol. 99, no. 3, pp. 466-467, 1942.

[26] H. Blumenfeld, "From molecules to networks: cortical/subcortical interactions in the pathophysiology of idiopathic generalized epilepsy," Epilepsia, vol. 44, no. S2, pp. 7-15, 2003.

[27] C. Luo, Y. Zhang, W. Cao et al., "Altered structural and functional feature of striato-cortical circuit in benign epilepsy with centrotemporal spikes," International Journal of Neural Systems, vol. 25, no. 06, p. 1550027, 2015.

[28] J. Ashburner, "A fast diffeomorphic image registration algorithm," NeuroImage, vol. 38, no. 1, pp. 95-113, 2007.

[29] J. Ashburner and K. J. Friston, "Voxel-based morphometrythe methods," NeuroImage, vol. 11, no. 6, pp. 805-821, 2000.

[30] M. W. Mosconi, S. Mohanty, R. K. Greene, E. H. Cook, D. E. Vaillancourt, and J. A. Sweeney, "Feedforward and feedback motor control abnormalities implicate cerebellar dysfunctions in autism spectrum disorder," The Journal of Neuroscience, vol. 35, no. 5, pp. 2015-2025, 2015.

[31] M. Glickstein, J. G. May 3rd, and B. E. Mercier, "Corticopontine projection in the macaque: the distribution of labelled cortical cells after large injections of horseradish peroxidase in the pontine nuclei," The Journal of Comparative Neurology, vol. 235, no. 3, pp. 343-359, 1985.

[32] J. D. Schmahmann, "From movement to thought: anatomic substrates of the cerebellar contribution to cognitive processing," Human Brain Mapping, vol. 4, no. 3, pp. 174-198, 1996.

[33] M. Glickstein and K. Doron, "Cerebellum: connections and functions," Cerebellum, vol. 7, no. 4, pp. 589-594, 2008.

[34] R. P. Dum and P. L. Strick, "An unfolded map of the cerebellar dentate nucleus and its projections to the cerebral cortex," Journal of Neurophysiology, vol. 89, no. 1, pp. 634-639, 2003.

[35] C. Mediavilla, F. Molina, and A. Puerto, "Retention of concurrent taste aversion learning after electrolytic lesioning of the interpositus-dentate region of the cerebellum," Brain Research, vol. 868, no. 2, pp. 329-337, 2000.

[36] L. Wang, F. Zou, Y. Shao et al., "Disruptive changes of cerebellar functional connectivity with the default mode network in schizophrenia," Schizophrenia Research, vol. 160, no. 1-3, pp. 67-72, 2014.

[37] J. D. Schmahmann, "The role of the cerebellum in cognition and emotion: personal reflections since 1982 on the dysmetria of thought hypothesis, and its historical evolution from theory to therapy," Neuropsychology Review, vol. 20, no. 3, pp. 236$260,2010$.

[38] W. Penfield and K. Welch, "The supplementary motor area of the cerebral cortex; a clinical and experimental study," AMA Archives of Neurology \& Psychiatry, vol. 66, no. 3, pp. 289$317,1951$.

[39] D. J. Serrien, L. H. A. Strens, A. Oliviero, and P. Brown, "Repetitive transcranial magnetic stimulation of the supplementary motor area (SMA) degrades bimanual movement control in humans," Neuroscience Letters, vol. 328, no. 2, pp. 89-92, 2002.

[40] A. D. Norden and H. Blumenfeld, "The role of subcortical structures in human epilepsy," Epilepsy \& Behavior, vol. 3, no. 3, pp. 219-231, 2002.

[41] Q. Li, C. Luo, T. Yang et al., "EEG-fMRI study on the interictal and ictal generalized spike-wave discharges in patients with childhood absence epilepsy," Epilepsy Research, vol. 87, no. 2-3, pp. 160-168, 2009.

[42] C. Luo, Q. Li, Y. Xia et al., "Resting state basal ganglia network in idiopathic generalized epilepsy," Human Brain Mapping, vol. 33, no. 6, pp. 1279-1294, 2012.

[43] A. M. Chilosi, P. Brovedani, M. Moscatelli, P. Bonanni, and R. Guerrini, "Neuropsychological findings in idiopathic occipital lobe epilepsies," Epilepsia, vol. 47, no. s2, pp. 76-78, 2006.

[44] P. Wolf and R. Goosses, "Relation of photosensitivity to epileptic syndromes," Journal of Neurology, Neurosurgery, and Psychiatry, vol. 49, no. 12, pp. 1386-1391, 1986.

[45] J. Townsend, E. Courchesne, J. Covington et al., "Spatial attention deficits in patients with acquired or developmental cerebellar abnormality," The Journal of Neuroscience, vol. 19, no. 13, pp. 5632-5643, 1999.

[46] J. D. Schmahmann and J. C. Sherman, "The cerebellar cognitive affective syndrome," Brain, vol. 121, no. 4, pp. 561-579, 1998.

[47] A. Baddeley, "Working memory," Science, vol. 255, no. 5044, pp. 556-559, 1992.

[48] S. J. Gilbert and P. W. Burgess, "Executive function," Current Biology, vol. 18, no. 3, pp. R110-R114, 2008.

[49] J. Ellis, Prospective Memory or the Realization of Delayed Intentions: A Conceptual Framework for Research, 1996. 
[50] M. E. Lancman, J. J. Asconapé, and J. K. Penry, "Clinical and EEG asymmetries in juvenile myoclonic epilepsy," Epilepsia, vol. 35, no. 2, pp. 302-306, 1994.

[51] D. W. Choi, "Glutamate receptors and the induction of excitotoxic neuronal death," Progress in Brain Research, vol. 100, pp. 47-51, 1994.

[52] A. Doble, "The role of excitotoxicity in neurodegenerative disease: implications for therapy," Pharmacology \& Therapeutics, vol. 81, no. 3, pp. 163-221, 1999.

[53] T. A. Jerde, E. P. Merriam, A. C. Riggall, J. H. Hedges, and C. E. Curtis, "Prioritized maps of space in human frontoparietal cortex," The Journal of Neuroscience, vol. 32, no. 48, pp. 17382-17390, 2012.

[54] S. L. Bressler, W. Tang, C. M. Sylvester, G. L. Shulman, and M. Corbetta, "Top-down control of human visual cortex by frontal and parietal cortex in anticipatory visual spatial attention," The Journal of Neuroscience, vol. 28, no. 40, pp. 10056-10061, 2008.

[55] N. Wenderoth, F. Debaere, S. Sunaert, and S. P. Swinnen, "The role of anterior cingulate cortex and precuneus in the coordination of motor behaviour," The European Journal of Neuroscience, vol. 22, no. 1, pp. 235-246, 2005.

[56] D. Sridharan, D. J. Levitin, and V. Menon, "A critical role for the right fronto-insular cortex in switching between central-executive and default-mode networks," Proceedings of the National Academy of Sciences of the United States of America, vol. 105, no. 34, pp. 12569-12574, 2008.

[57] A. Jakab, P. P. Molnár, P. Bogner, M. Béres, and E. L. Berényi, "Connectivity-based parcellation reveals interhemispheric differences in the insula," Brain Topography, vol. 25, no. 3, pp. 264-271, 2012.

[58] J. N. Giedd, M. Keshavan, and T. Paus, "Why do many psychiatric disorders emerge during adolescence?," Nature Reviews Neuroscience, vol. 9, no. 12, pp. 947-957, 2008.

[59] R. Kuruba, B. Hattiangady, and A. K. Shetty, "Hippocampal neurogenesis and neural stem cells in temporal lobe epilepsy," Epilepsy \& Behavior, vol. 14, no. 1, pp. 65-73, 2009.

[60] J. R. Binder, R. H. Desai, W. W. Graves, and L. L. Conant, "Where is the semantic system? A critical review and metaanalysis of 120 functional neuroimaging studies," Cerebral Cortex, vol. 19, no. 12, pp. 2767-2796, 2009.

[61] E. Marder and J. M. Goaillard, "Variability, compensation and homeostasis in neuron and network function," Nature Reviews Neuroscience, vol. 7, no. 7, pp. 563-574, 2006.

[62] P. F. Qiao and G. M. Niu, "Resting-state fMRI findings in patients with first-episode idiopathic epilepsy before and after treatment," Neurosciences (Riyadh), vol. 22, no. 4, pp. 316-319, 2017. 


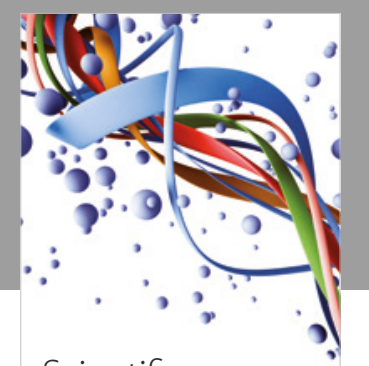

Scientifica
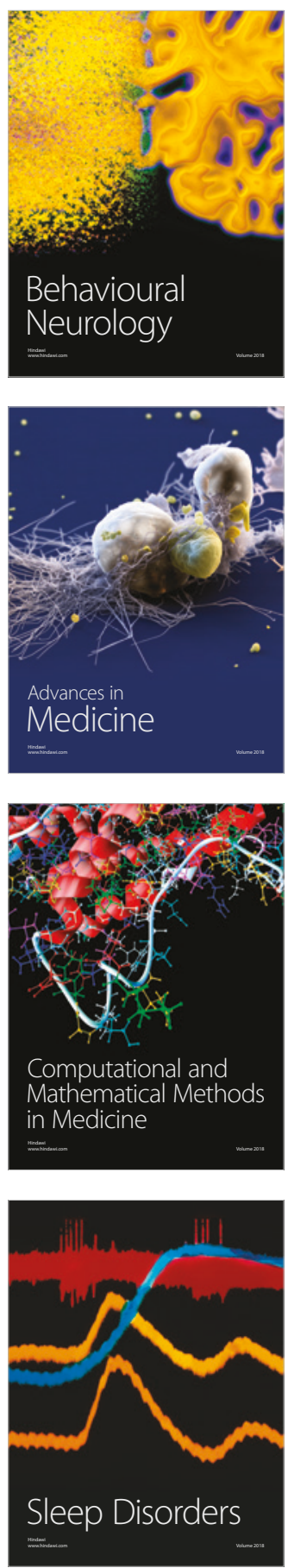

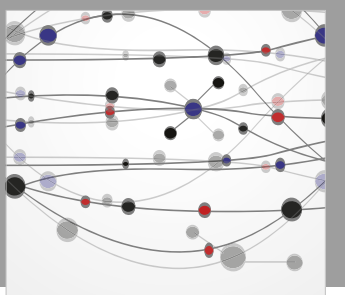

The Scientific World Journal

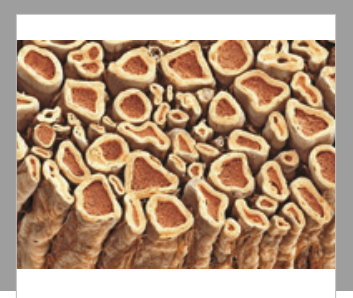

Case Reports in

Neurological Medicine

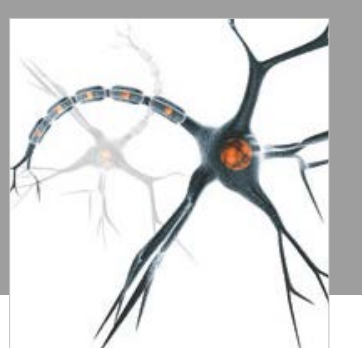

Neural Plasticity

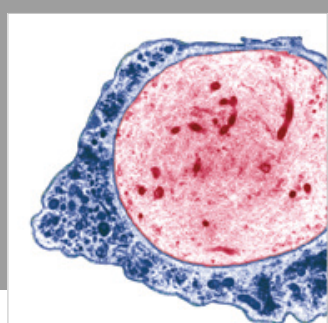

Multiple Sclerosis

International

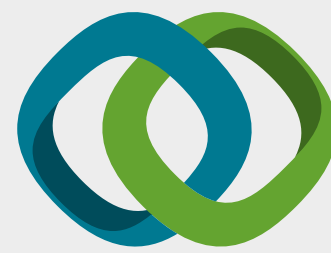

Hindawi

Submit your manuscripts at

www.hindawi.com
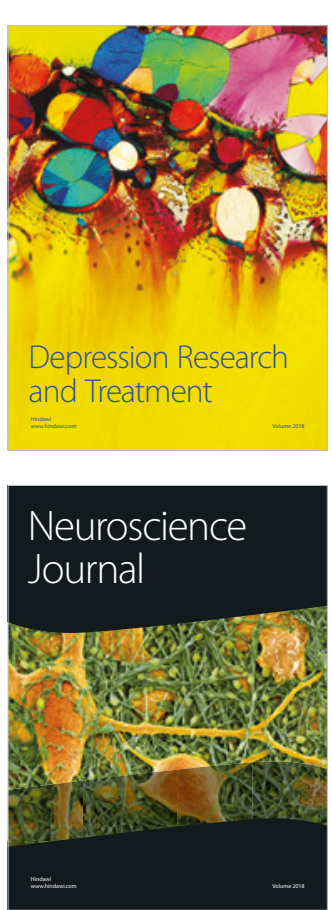

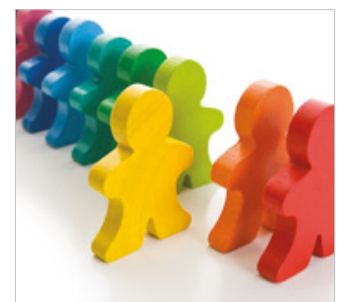

Autism

Research and Treatment
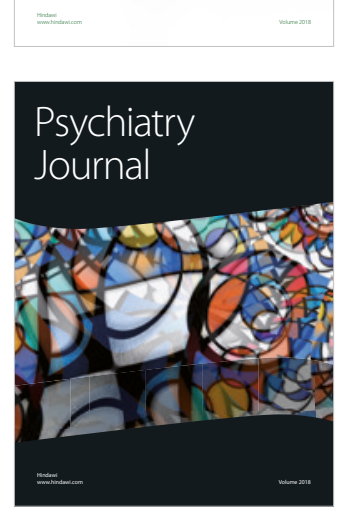
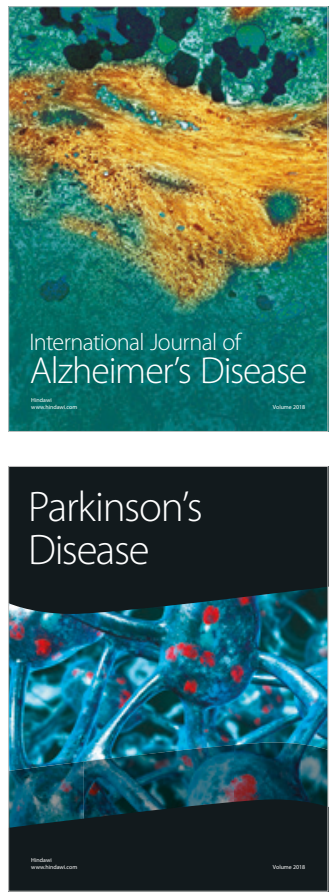
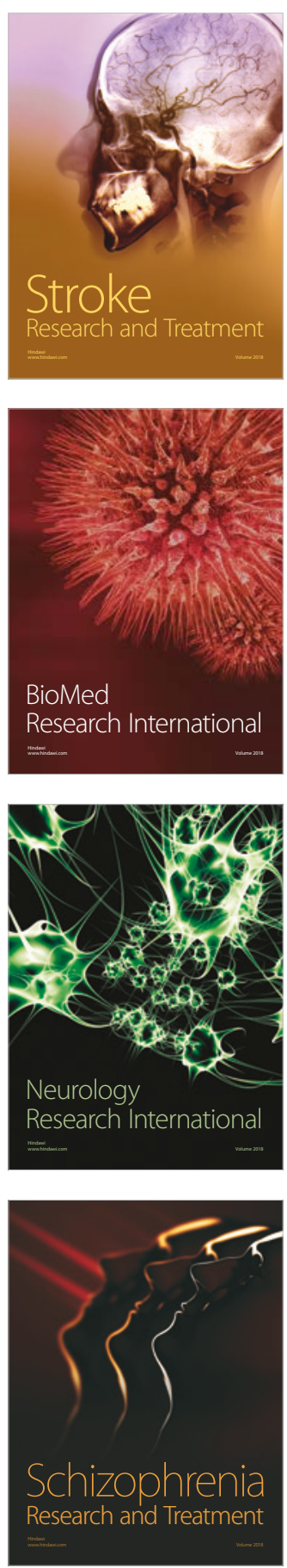